\title{
Pulmonary Embolism in COVID-19 and the Unanswered Questions
}

\author{
Anchit Bharat $^{\mathrm{a}, \mathrm{d}}$, Nikita Jain ${ }^{\mathrm{b}}$, Veerpal Singh ${ }^{\mathrm{c}}$
}

\begin{abstract}
Severe acute respiratory syndrome coronavirus 2 (SARS-CoV-2) has ravaged the global society as we know it. As almost a dozen pharmaceutical agents go into randomized controlled clinical trials, multiple studies have surfaced trying to associate a hypercoagulable state to coronavirus disease 2019 (COVID-19) patients. We report two COVID-19 cases who presented with occlusive pulmonary embolism (PE) strongly supporting a hypercoagulable state incurred by SARS-CoV-2. This is significant as it is one of the early reports of such an initial presentation of COVID-19 in the USA. Through our report, we invite the medical community to share a perspective about long-term management guidelines for SARS-CoV-2 associated venous thromboembolism (VTE) and prompt future research.
\end{abstract}

Keywords: SARS-CoV-2; COVID-19; Pulmonary embolism; Venous thromboembolism

\section{Introduction}

Severe acute respiratory syndrome coronavirus 2 (SARSCoV-2) has ravaged the global society as we know it. The medical community is in a desperate need to understand the pathophysiology of coronavirus disease 2019 (COVID-19) (caused by SARS-CoV-2) and explore possible therapeutic strategies in the wake of widespread mortality. At the time of writing this article, globally, 305,395 people have succumbed to this disease with 4,508,435 total infections. As almost a dozen pharmaceutical agents go into randomized controlled clinical trials, multiple studies have surfaced try-

Manuscript submitted May 4, 2020, accepted May 16, 2020

Published online May 28, 2020

andiana University Health Ball Memorial Hospital, Muncie, IN, USA

${ }^{b}$ Chicago Medical School, Rosalind Franklin University of Medicine and Sciences, Mchenry, IL, USA

'Northwestern Medicine Hospital, Mchenry, IL, USA

${ }^{\mathrm{d} C o r r e s p o n d i n g ~ A u t h o r: ~ A n c h i t ~ B h a r a t, ~ I n d i a n a ~ U n i v e r s i t y ~ H e a l t h ~ B a l l ~ M e m o-~}$ rial Hospital, 4201 W University Avenue, Muncie, IN 47303, USA.

Email: anchitbharat92@gmail.com

doi: https://doi.org/10.14740/jmc3488 ing to associate a hypercoagulable state to COVID-19 patients [1]. We reference these studies and present two novel COVID-19 cases who presented with pulmonary embolism (PE).

\section{Case Report}

The summary of cases with pertinent patient characteristics, histories, findings and supporting data are presented in Table 1. Until now, there have been only sporadic cases of occlusive PE in COVID-19 patients, coming from China, Italy and the USA. Both our patients presented with dyspnea preceded by a mild flu-like illness and were found to have pulmonary emboli with significant clot burden at initial presentation. There were no significant predisposing risk factors, other than a hypercoagulable state due to COVID-19. The history of prostate carcinoma in patient 2 is unlikely to be contributory as he was in remission, suggested by a normal prostatespecific antigen (PSA). Both patients were treated with heparin products. Patient 1 remains hospitalized with moderate illness (day 4). Patient 2 required 3 days of inpatient stay and was discharged on newer oral anticoagulants (NOACs) with a recommended duration of 3 months. Hypercoagulable workup including factor V Leiden mutation, protein $\mathrm{C}$, protein S, antithrombin III levels, and anti-phospholipid antibody tests was ordered upon outpatient follow-up, which remains pending.

\section{Discussion}

Initial studies from Greece and France endorse that COVID-19 patients might be at a higher risk of developing venous thromboembolism (VTE), which has been attributed to a hypercoagulable state [1]. The exact putative mechanism of this observation still remains elusive, but the role of antiphospholipid antibodies (APLAs) has been implicated [2]. Interesting autopsy findings from China suggest hyaline thrombi in some microvessels of the alveolar septum on immunohistochemical staining [3]. Our European and Chinese colleagues recommend an early initiation of anticoagulation therapy in selected patients $[1,4]$. This has shown to improve prognosis of this group of COVID-19 patients [5]. In summary, all these studies point towards a similar direction, decoding the labyrinth, i.e. pathophysiology of COVID-19 and how anticoagulation 
Table 1. Pertinent Patient Characteristics, Histories, Laboratory Findings, and Imaging Results

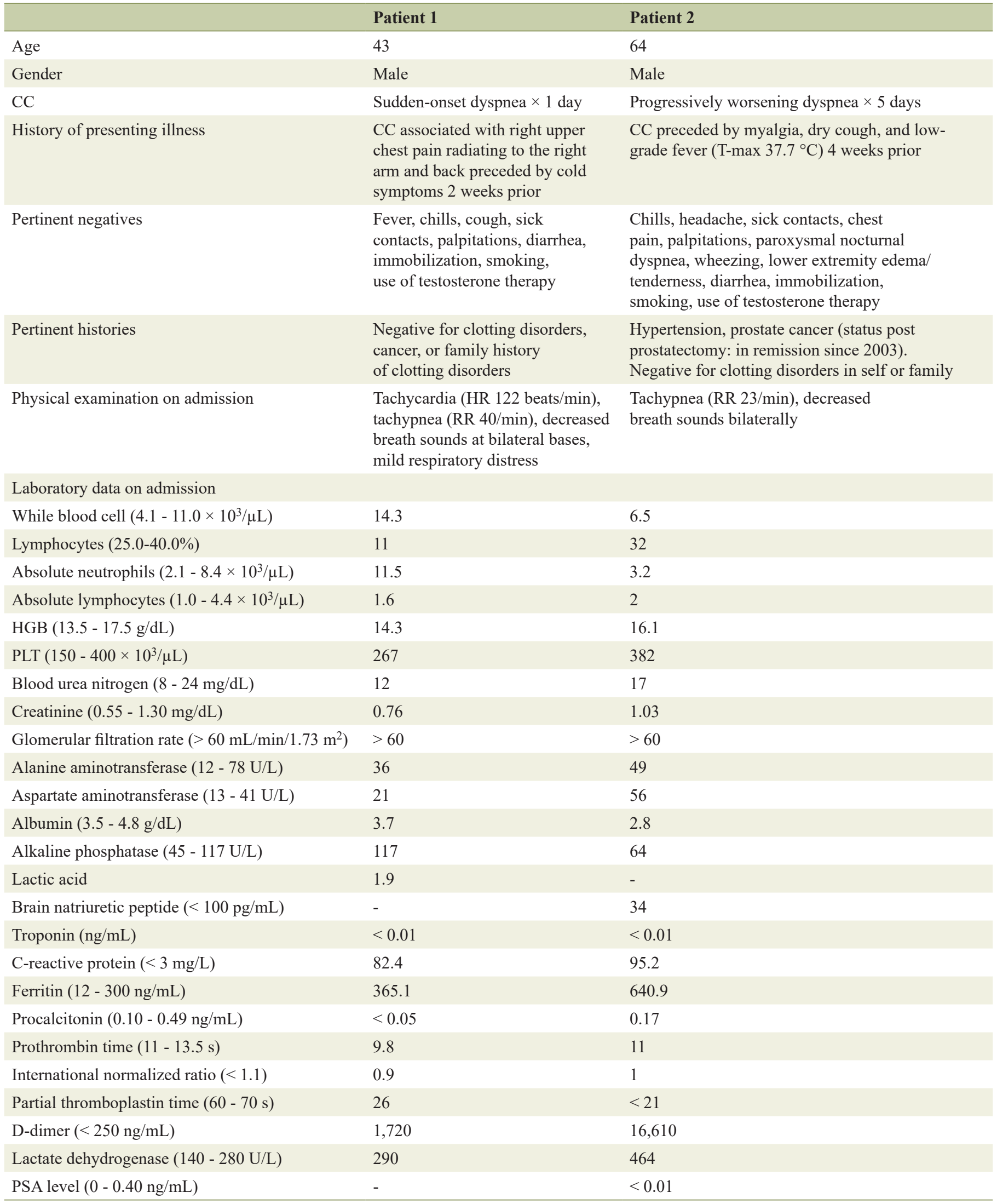


Table 1. Pertinent Patient Characteristics, Histories, Laboratory Findings, and Imaging Results - (continued)

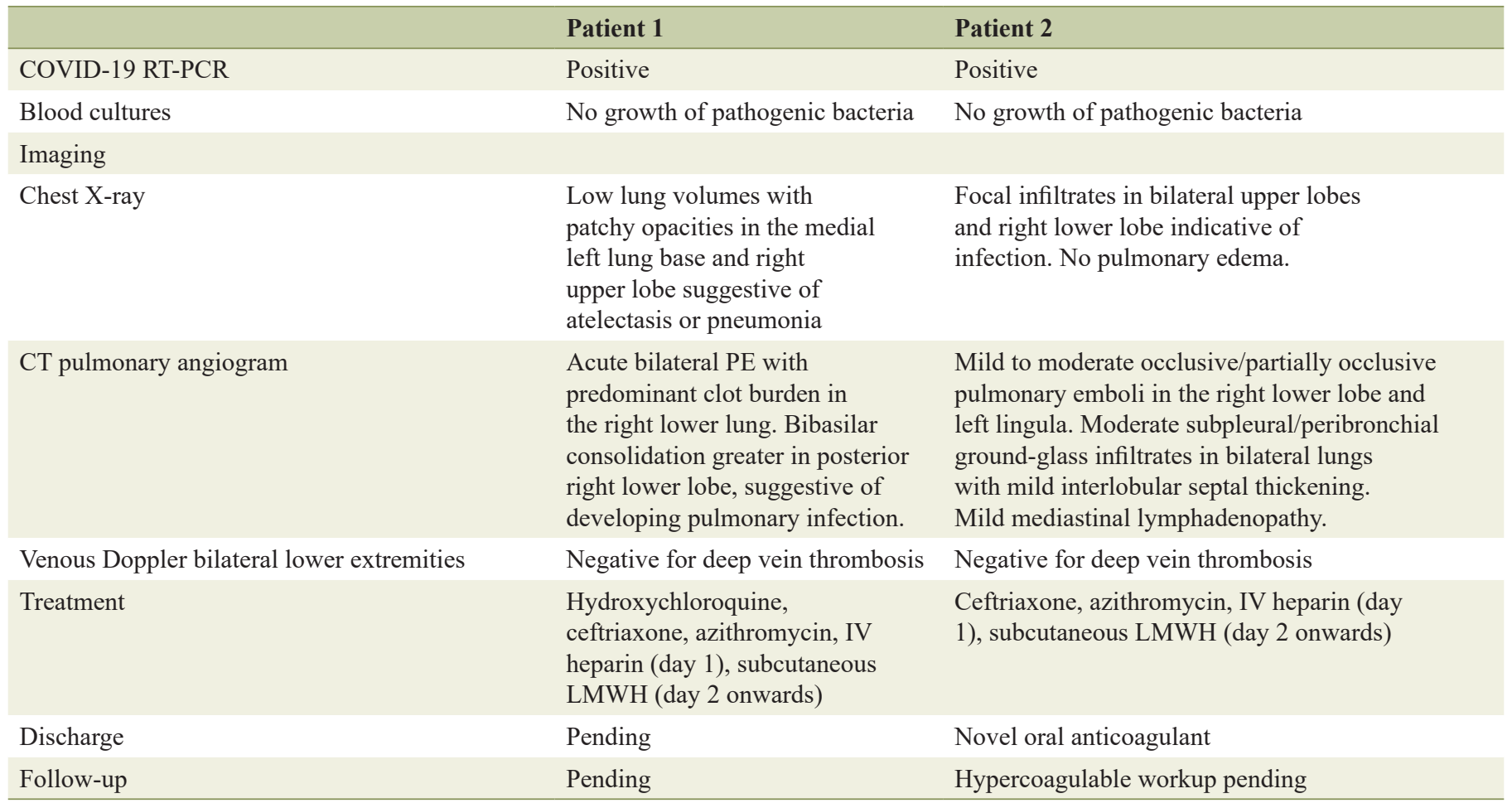

CC: chief complaint; HR: heart rate; RR: respiratory rate; HGB: hemoglobin; PLT: platelet; PSA: prostate-specific antigen; COVID-19: coronavirus disease 2019; RT-PCR: reverse-transcriptase polymerase chain reaction; CT: computerized tomography; IV: intravenous; LMWH: low-molecular-weight heparin.

therapy might fit in the algorithm. Future randomized controlled studies might help clear this association. In the meantime, low-molecular-weight heparin (LMWH) is being widely used in patients who fit this picture $[4,5]$. This brings us to our questions to the medical community: 1) Are NOACs appropriate for these patients? 2) Should we consider these PEs as provoked or unprovoked? 3) What should be the duration of therapy?

\section{Acknowledgments}

None to declare.

\section{Financial Disclosure}

None to declare.

\section{Conflict of Interest}

None to declare.

\section{Informed Consent}

Verbal consent for publication of the clinical details was ob- tained from both patients and all patient identifiable information is anonymized in the manuscript.

\section{Author Contributions}

$\mathrm{AB}$ and $\mathrm{NJ}$ contributed to literature review and manuscript preparation; VS contributed to case information and reviewed manuscript. All authors have read the uploaded manuscript and consented to submission.

\section{Data Availability}

The authors declare that data supporting the findings of this study are available within the article. Any further inquiries regarding supporting data availability of this study can be directed to the submitting author.

\section{Abbreviations}

SARS-CoV-2: severe acute respiratory syndrome coronavirus 2; COVID-19: coronavirus disease 2019; PE: pulmonary embolism; VTE: venous thromboembolism; APLAs: antiphospholipid antibodies; LMWH: low-molecular-weight heparin; NOAC: newer oral anticoagulant; RT-PCR: reverse-transcriptase polymerase chain reaction; PSA: prostate-specific an- 
tigen; HR: heart rate; RR: respiratory rate; HGB: hemoglobin; PLT: platelet; CT: computerized tomography; IV: intravenous

\section{References}

1. Terpos E, Ntanasis-Stathopoulos I, Elalamy I, Kastritis E, Sergentanis TN, Politou M, Psaltopoulou T, et al. Hematological findings and complications of COVID-19. Am J Hematol. 2020.

2. Zhang Y, Xiao M, Zhang S, Xia P, Cao W, Jiang W, Chen $\mathrm{H}$, et al. Coagulopathy and antiphospholipid antibodies in patients with COVID-19. N Engl J Med. 2020;382(17):e38.

3. Yao XH, Li TY, He ZC, Ping YF, Liu HW, Yu SC, Mou $\mathrm{HM}$, et al. [A pathological report of three COVID-19 cases by minimal invasive autopsies]. Zhonghua Bing Li Xue Za Zhi. 2020;49(5):411-417.

4. Zhang Y, Cao W, Xiao M, Li YJ, Yang Y, Zhao J, Zhou $\mathrm{X}$, et al. [Clinical and coagulation characteristics of $7 \mathrm{pa}-$ tients with critical COVID-2019 pneumonia and acro-ischemia]. Zhonghua Xue Ye Xue Za Zhi. 2020;41(0):E006.

5. Tang N, Bai H, Chen X, Gong J, Li D, Sun Z. Anticoagulant treatment is associated with decreased mortality in severe coronavirus disease 2019 patients with coagulopathy. J Thromb Haemost. 2020;18(5):1094-1099. 